\title{
Utilización de los objetos de aprendizaje y su relación en el rendimiento académico de la asignatura de Física I
}

Use of learning objects and their relationship in the academic performance of the subject of Physics I

Juan Manuel Martinez Nogales . ${ }^{1}$, Jorge Cachuput Gusñay. ${ }^{2}$, Jaime Rodrigo Guilcapi Mosquera ${ }^{3}$ \& Luis Fernando Carrasco Pilco ${ }^{4}$

\begin{abstract}
.
The use of learning objects and its relation in the academic performance of the signature of Physics I in the students of the first semester of the Faculty of Engineering of Universidad Nacional de Chimborazo. The low performance reached by students in Physics due to the exigent level of university training produces difficulties in the conceptual domain, as well as methods for its study. This work aims to determine the relation between using learning objects and the academic performance in the study of the Dynamics of the particle. The research is of correlational type and quasi- experimental design, the proposed methodology is based on an instructional design model that guides the pedagogics part which recommends the use of temples. Taking into account the main actors at taking the statistics test ( $\mathrm{z}$ ) for independent samples it is shown that the groups of students who used learning objects got a better academic performance than students didn't do it. Evolution of the learning outcomes in each student is very evident. OA bettered the academic performance in the students of Engineering who take the signature of Physics related to study of the Dynamics of the particle; OA improves the teaching-learning process and academic performance including every single student in collaborative and cooperative work.
\end{abstract}

Keywords: Dynamics of the particle, students in Physics, used learning objects, the academic performance.

\footnotetext{
1 Escuela Superior Politécnica de Chimborazo, Facultad de Recursos Naturales. Riobamba, Ecuador, jumartinez@espoch.edu.ec

${ }^{2}$ Escuela Superior Politécnica de Chimborazo, Facultad de Recursos Naturales. Riobamba, Ecuador. jcachuput@espoch.edu.ec

${ }^{3}$ Universidad Técnica de Ambato, Ingeniería en Telecomunicaciones, Ambato, Ecuador, jr.guilcapi@uta.edu.ec

${ }^{4}$ Colegio Internacional Rudolf Steiner. Quito, Ecuador. ferchoone2003@yahoo.com
} 


\section{Resumen.}

La utilización de los objetos de aprendizaje y su relación en el rendimiento académico de la asignatura de física I, en los estudiantes de primer semestre de la facultad de ingeniería de la Universidad Nacional de Chimborazo. El bajo rendimiento que el estudiante alcanza en física, por el nivel de exigencia que se aplica en la formación universitaria, generan dificultades en el dominio conceptual, así como en la apropiación de los métodos para su estudio, este trabajo propone, determinar la relación entre la utilización de los objetos de aprendizaje y el rendimiento académico en el estudio de la Dinámica de la partícula, el cual se realizó mediante una investigación de tipo correlacional y diseño Cuasi-experimental, la metodología propuesta se basó en el modelo de Diseño Instruccional que guie en la parte pedagógica en la que se recomienda el uso de algunas plantillas. Tomando en cuenta a los actores principales, como se puede apreciar, al realizar la prueba estadística de (z) para muestras independientes, se puede ver que efectivamente el grupo de estudiantes que utilizan los objetos de aprendizaje obtienen un rendimiento académico significativamente superior en comparación con los estudiantes que no usaron los objetos de aprendizaje. En la que se nota claramente la evolución de los logros de aprendizaje en cada uno de los estudiantes. La incorporación de los OA mejoró el rendimiento académico en los estudiantes de ingeniería que cursan la asignatura de física I en el estudio de la dinámica de la partícula, los OA mejoran el proceso de enseñanza- aprendizaje y elevan el rendimiento académico, involucrando a todos los estudiantes a un trabajo colaborativo y cooperativo.

Palabras claves: Objetos de aprendizaje, rendimiento académico, metodología, dinámica de la partícula

\section{Introducción.}

La educación es un fenómeno universal necesario para la continuidad cultural de la humanidad a través de la cual las generaciones antecedentes preparan a las siguientes, en un continuo de transferencia de informaciones, conocimientos y habilidades.

De esa manera, las demandas sociales en cierto momento histórico-social determinan los objetivos de la educación y estos se caracterizan en los contenidos y están estrechamente unidos a los métodos a utilizar en la enseñanza. Así, las Instituciones de Enseñanza y la sociedad son indivisibles " la sociedad vive y se desarrolla tal como aprende y aprende tal como quiere vivir" (Davidov, 1991, pág. 118; Antueno, Eduardo A. , 2008) .

Los Objetos de aprendizaje permiten al estudiante modificar algún parámetro y observar el efecto que produce en su rendimiento académico, mediante las simulaciones que proveen una representación interactiva de la realidad que permite al estudiante probar y descubrir cómo funciona y se comporta un fenómeno, el uso de este tipo de herramientas motiva al estudiante para que pueda desarrollar y lograr una mejor comprensión.

Para evitar la falta de interés y la repetición de conceptos de los estudiantes en la asignatura de Física I de manera particular en el estudio de la Dinámica de la partícula, es necesario utilizar los Objetos de Aprendizaje apoyados con los simuladores Interactive Physics y el 
EJS Java, con la finalidad de aclarar conceptos y ayudar a resolver problemas en los que se presentan una mayor dificultad, mejorando la calidad de la educación, contribuyendo al estudiante a que desarrollen mejor sus capacidades habilidades y destrezas en especial su capacidad creativa.

Con el fin de que se cumplan con los objetivos de la Universidad y la Facultad de Ingeniería beneficiaria de este proyecto, de manera especial el primer semestre de la carrera de Arquitectura donde aún no se han realizado estudios semejantes en el área de Física.

\section{Problema}

El problema de esta investigación es: ¿De qué manera incide la utilización de los objetos del aprendizaje en el rendimiento académico en el estudio de la Dinámica de la partícula correspondiente a la asignatura de Física I, en los estudiantes del primer semestre de la Facultad de Ingeniería de la Universidad Nacional de Chimborazo?

\section{Objetivos del estudio}

Específicamente, respecto a la ciencia Física, esta es una ciencia que necesita particularmente del razonamiento deductivo para su comprensión y acostumbradamente se ve como una asignatura de asimilación difícil por parte de los estudiantes del área tecnológica. Lo que se desea comprobar es que gran parte de esta dificultad no está en la complejidad de la ciencia en sí misma, sino en el hecho de que los estudiantes no desarrollaron apropiadamente la formación de los procedimientos lógicos del pensamiento, así como, la enseñanza de la Física no haberse desarrollado científicamente con métodos elaborados, capaces de formar tales procedimientos.

Los objetivos del presente trabajo son: a) Utilizar los Objetos de Aprendizaje para mejorar el Rendimiento Académico en el estudio de la Dinámica de la partícula, de la asignatura de Física I; b) Construir los objetos de aprendizaje que permitan mejora el rendimiento académico; c) Incorporar los objetos de aprendizaje dentro del proceso educativo; d) Proponer una metodología para su incorporación de los objetos de aprendizaje dentro del proceso de enseñanza aprendizaje.

\section{Rendimiento académico}

En la vida académica, habilidad y esfuerzo no son sinónimos; el esfuerzo no garantiza un éxito, y la habilidad empieza a cobrar mayor importancia. Esto se debe a cierta capacidad cognitiva que le permite al alumno hacer una elaboración mental de las implicaciones causales que tiene el manejo de las autopercepciones de habilidad y esfuerzo. Dichas autopercepciones, si bien son complementarias, no presentan el mismo peso para el estudiante; de acuerdo con el modelo, percibirse como hábil (capaz) es el elemento central. De acuerdo con lo anterior se derivan tres tipos de estudiantes según (Covington, The motive for self-worth., 1984) .

a) "Los orientados al dominio. Sujetos que tienen éxito escolar, se consideran capaces, presentan alta motivación de logro y muestran confianza en sí mismos. 
b) Los que aceptan el fracaso. Sujetos derrotistas que presentan una imagen propia deteriorada y manifiestan un sentimiento de desesperanza aprendido, es decir que han aprendido que el control sobre el ambiente es sumamente difícil o imposible, y por lo tanto renuncian al esfuerzo.

c) Los que evitan el fracaso. Aquellos estudiantes que carecen de un firme sentido de aptitud y autoestima y ponen poco esfuerzo en su desempeño; para "proteger" su imagen ante un posible fracaso, recurren a estrategias como la participación mínima en el salón de clases, retraso en la realización de una tarea, trampas en los exámenes, etc. "

Cuando se invierte mucho esfuerzo no se ve el verdadero nivel de habilidad, de tal forma que esto no amenaza la estima o valor como estudiante, y en tal caso, el sentimiento de orgullo y la satisfacción son grandes. (Tejedor F. y., 2007)

Lo anterior significa que, en una situación de éxito, las autopercepciones de habilidad y esfuerzo no perjudican ni dañan la estima ni el valor que el profesor otorga. Sin embargo, cuando la situación es de fracaso, las cosas cambian.

Dado que una situación de fracaso pone en duda su capacidad, es decir, su autovaloración, algunos estudiantes evitan este riesgo, y para ello emplean ciertas estrategias como la excusa y manipulación del esfuerzo, con el propósito de desviar la implicación de inhabilidad (Covington \& Omelich, Journal Personality and Social Psychology, 1979)

El empleo desmedido de estas estrategias trae como consecuencia un deterioro en el aprendizaje, se está propenso a fracasar y se terminará haciéndolo tarde o temprano (Covington, 1984), lo que en forma análoga nos recuerda el `efecto Pigmalión`en el proceso educativo, es decir, una profecía de fracaso escolar que es auto-cumplida.

Resulta evidente, que el abordaje del rendimiento académico no podría agotarse a través del estudio de las percepciones de los estudiantes sobre las variables habilidad y esfuerzo, así como tampoco podría ser reducida a la simple comprensión entre actitud y aptitud del estudiante.

El rendimiento académico de los estudiantes universitarios constituye un factor importante y fundamental para la evaluación de la calidad educativa. Es la suma de los diferentes y complejos factores que intervienen en el estudiante, $\mathrm{y}$ ha sido definido con un valor levantado al logro de estudiante en el cumplimiento de las tareas académicas, se mide mediante las calificaciones alcanzadas, con una valoración cuantitativa, cuyos resultados indican las asignaturas aprobadas o reprobadas, la deserción y el grado de éxito académico Pérez, Ramón, Sánchez (2000).

Cada universidad establece sus propios criterios evaluativos para obtener una ponderación de las asignaturas que cursa cada estudiante, donde se toma en cuenta número de asignaturas, número de créditos y el valor obtenido en cada una de ellas que se conoce como record académico. Se toma las calificaciones como la medida de enseñanza teniendo en cuenta que son producto de los condicionantes como de tipo personal del estudiante, como didácticas del docente. (Tejedor f. y., 2007) 
La función del docente es enlazar procesos de construcción del estudiante, que se ha convertido en una preocupación del docente porque los estudiantes conozcan hechos científicos, pensando que los estudiantes aprenden por repetición las mismas que van a depender siempre del docente. (Ruiz, 2005).

\section{Metodologia.}

\section{Tipo de investigación}

La presente es una investigación explicativa y correlacional, pues busca determinar las causas y los factores del rendimiento académico, al utilizar los objetos de aprendizaje, así como el nivel de correlación entre las dos variables de estudio. (Urquizo, 2005).

\section{Diseño de la investigación}

El diseño de la investigación registrada en el documento presente es cuasi experimental (Urquizo, 2005)

\section{Métodos de investigación}

Se aplicarán los siguientes métodos: Científico e hipotético deductivo en todo el estudio por la epistemología de las ciencias experimentales; inductivo deductivo en la elaboración del marco teórico de la tesis; analítico sintético tanto en la tabulación de datos cuanto en la aplicación metodológica y estadístico en la reducción positivista de las variables de la investigación; otros métodos son los aplicados a la generalidad del estudio; como es el ecléctico y metodológicamente el piramidal.

\section{Técnicas e instrumentos para la recolección de datos}

Las técnicas e instrumentos de acopio de datos se validan pues miden lo que deben medir sin provocar sesgos de apreciación; incluyen la observación no estructurada; las encuestas de registro de aprendizajes, las pruebas estructuradas individuales; las matrices de registro de indicadores piramidales; los trabajos grupales, trabajos individuales, exámenes oficiales y lecciones.

Entre las técnicas aplicadas a esta investigación con fines de acopio de datos que permitan una interpretación confiable de la aplicación de los objetos de aprendizaje son las siguientes: observación no estructurada y cuestionarios objetivos con preguntas cerradas de corte positivista cuantitativo. El cuadro siguiente describe técnicas e instrumentos utilizados para el efecto.

Tabla No 1Técnicas e instrumentos de recolección de información

\begin{tabular}{ll}
\hline TECNICAS & INSTRUMENTOS \\
\hline Encuesta & Cuestionario \\
Test & Prueba Objetiva \\
Observación & Fichas o guías de observación \\
\hline
\end{tabular}




\section{Población y muestra}

Se trabajó con dos grupos de estudiantes del primer semestre de la Facultad de Ingeniería de la Carrera de arquitectura 36 estudiantes del paralelo "B" grupo de experimentación y 32 estudiantes del paralelo " $\mathrm{A}$ " grupo de control.

Con el primer semestre paralelo "A" se trató el tema Dinámica de la partícula con métodos tradicionales y el primer semestre paralelo " $\mathrm{B}$ " con objetos de aprendizaje, done lo estudiantes interactuaron en un EVA, en lo que les permitió interactuar y afianzar conocimientos, realizando un trabajo autónomo y cooperativo y colaborativo. El Tipo de muestreo utilizado para este trabajo de investigación será el MUESTREO ESTRATIFICADO

Tabla No 2 POBLACIÓN Y MUESTRA

\begin{tabular}{ccccc}
\hline Paralelos & Población & $\begin{array}{c}\text { Margen } \\
\text { de error } \\
(\boldsymbol{\%})\end{array}$ & $\begin{array}{c}\text { Nivel de } \\
\text { confianza } \\
(\boldsymbol{\%})\end{array}$ & Muestra \\
\cline { 2 - 5 } A & 37 & 10 & 90 & 32 \\
B & 42 & 10 & 90 & 36 \\
Total & $\mathbf{7 9}$ & & & $\mathbf{6 8}$ \\
\hline
\end{tabular}

$$
\mathbf{n}=\frac{\mathbf{Z}^{2} \cdot \mathbf{p} \cdot(\mathbf{1}-\mathbf{p})}{\cdot \mathbf{e}^{2}}
$$

$\mathrm{n}=$ tamaño de la muestra que queremos calcular $\mathrm{Z}=$ es la desviación del valor medio que se acepta para lograr el nivel de confianza, que tiene la distribución de Gauss Nivel de confianza $90 \% \geq Z=1,645$ $\mathrm{e}=$ margen de error máximo admitido $=$ $10 \%$

$\mathrm{p}=$ proporción que se espera encontrar, se recomienda el $50 \%$

La muestra considerada para este análisis será de 68 estudiantes, tomando en cuenta que el grupo experimental corresponde al $53 \%$ que equivale a 36 estudiantes y el grupo de control tomando el $47 \%$ que corresponde a 32 estudiantes.

\section{Simulaciones}

Es una técnica que se utiliza para analizar y estudiar sistemas complejos, permite reunir información pertinente sobre el comportamiento del sistema, porque ejecuta un modelo computarizado, sustentando que la simulación no es una técnica de optimización, más bien es una técnica que se utiliza para estimar las medidas de desempeño del sistema modelado, en este trabajo de investigación se ha utilizado simuladores de física como: Interactive Physics y EJS Java 


\section{Interactive Physics}

Es un programa que se utiliza para realizar simulaciones de movimientos físicos, principalmente de la Mecánica en especial dentro de lo que comprende a la Dinámica de la partícula, se puede calcular cantidades como la velocidad, desplazamiento, aceleración, tiempo, etc. Se puede controlar y modificar características físicas de un objeto como la gravedad, vectores, fuerza, etc.

Es un software fácil de utilizar y presenta un atractivo visual. Se utiliza para analizar virtualmente como se desplazan los cuerpos en una superficie inclinada sujetas a cuerdas que transmiten movimiento utilizando poleas, ejemplo.

En un sistema de cuerpos conectados, el coeficiente de fricción cinética es 0,20 bajo los cuerpos B y C. Determine la aceleración de cada cuerpo y la tensión en la cuerda que sostiene al cuerpo A.

Gráfico No 1. Simulación de cuerpos conectados

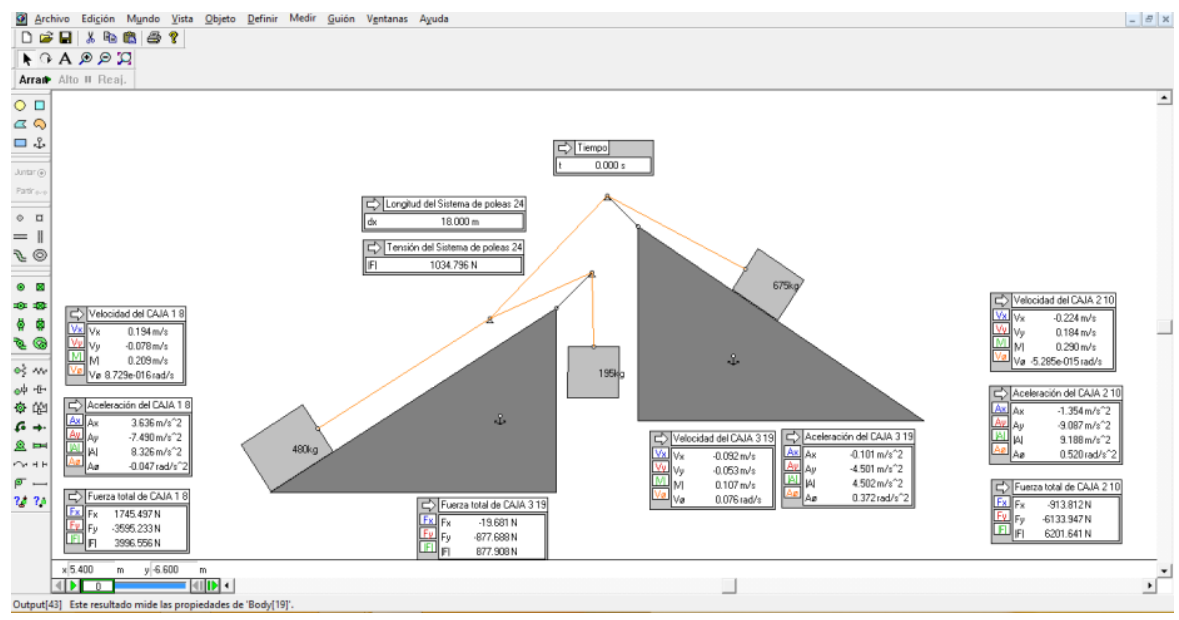

Easy Java Simulation (EJS)

Es un software libre y de código abierto, utiliza Java, multilenguaje, no requiere conocimientos avanzados de programación, lo pueden utilizar docentes y estudiantes, que saben programar bien y crear simulaciones "desde cero", permite también modificar ligeramente otras simulaciones.

¿Por qué utilizar EJS?

Por ser un buen software educativo y es:

Adoptable

Resuelve numéricamente o analíticamente

Simula bien un proceso

Modeliza las características que se necesita

Se puede modificar el modelo

Adaptable

Se puede modificar el modelo

Se puede visualizar cosas nuevas 
Se puede aumentar y restringir la capacidad de interacción del estudiante

Una buena parte de la investigación en física experimental y teórica no puede hacerse sin ayuda de ordenadores.

Gráfico No 2. Simulación de cuerpos conectados

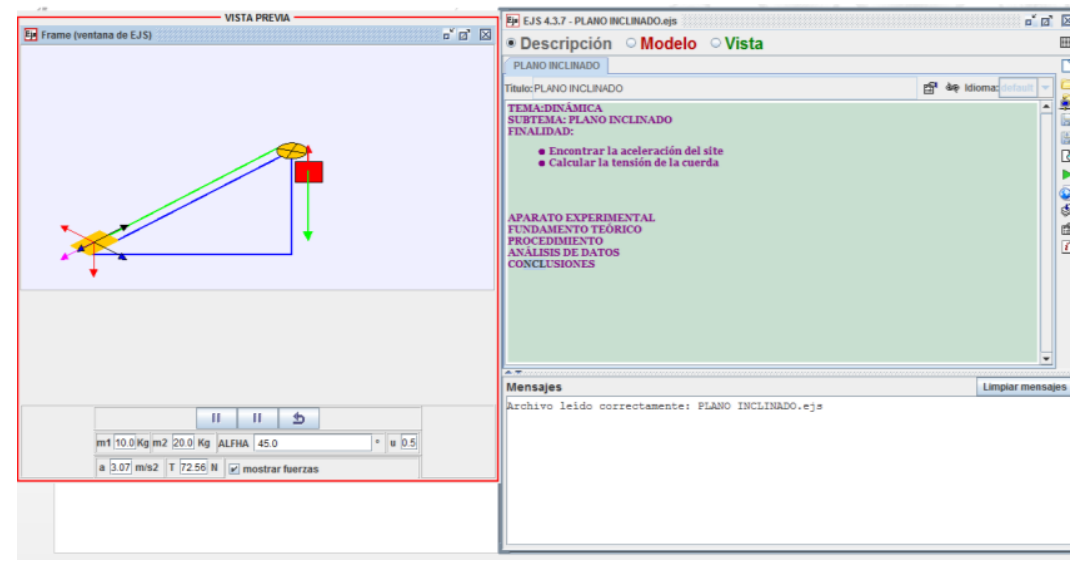

Evaluación del aprendizaje. Test, cuestionarios

Cuadro No 1 Matriz de evaluación

\begin{tabular}{lll}
\hline EVALUACIÓN & & \\
\hline No. & $\begin{array}{l}\text { No. } \\
\text { preguntas }\end{array}$ & Tipo \\
1 & 10 & Falso/Verdadero \\
2 & 10 & Opción Múltiple \\
\hline
\end{tabular}

\section{FASE 3. Nesarrollo}

Con la ayuda de las TIC's, se arma la estructura general del OA, propuesto en la fase del Diseño

\section{PASO 5. Armado}

La estructura general, de lo realizado en la fase anterior es necesario integrarlo en un archivo HTML. Con la siguiente matriz, Nombre de la institución Logo de la institución

Fase 4. Implantación 


\section{Rendimiento Académico}

Es la medida de las capacidades del estudiante, en lo que se expresa lo que, aprendido a lo largo del proceso formativo, también se conoce como la capacidad del estudiante para responder a los estímulos educativos.

Resumiendo, el rendimiento académico es un indicador del nivel de aprendizaje alcanzado por el estudiante, por ello, el sistema educativo brinda tanta importancia a dicho indicador. En tal sentido, el rendimiento académico se convierte en una "tabla imaginaria de medida" para el aprendizaje logrado en el aula, que constituye el objetivo central de la educación ¿Qué paso cuando utilizaron los Objetos de Aprendizaje?

Después que los estudiantes utilizaron los Objetos de aprendizaje se notó una mejoría en el nivel de aprendizaje, porque los estudiantes investigaban y preguntaban, superaron el temor de participar y pasar al frente a la pizarra.

Realizaron aplicaciones relacionados con hechos y situaciones prácticas de la carrera, se notó el cambio de actitud y gran motivación para trabajar en equipo, desarrollando sus propias aplicaciones relacionadas con la carrera, construyendo sus propios modelos y simulaciones.

\section{Resultados.}

\section{Análisis e interpretación de resultados}

Se presenta a continuación la matriz de resultados de la aplicación "Los Objetos de Aprendizaje (OA)y su relación en el Rendimiento Académico de la asignatura de Física I" en el grupo cuasi-experimental. Tomando en cuenta los siguientes parámetros:

$\mathrm{CD}=$ Componente de docencia; $\quad \mathrm{PAE}=$ Prácticas de Aplicación y Experimentación;

$\mathrm{AAA}=$ Actividades de aprendizaje autónomo

Análisis e interpretación de resultados

Tabla No 3 Matriz de rendimiento académico

\begin{tabular}{lcccc}
\hline & CD/4 & PAE/3 & AAA/3 & NOTA/10 \\
\hline $\begin{array}{c}\text { 1P-CON } \\
\text { OA }\end{array}$ & 1,24 & 2,03 & 2,2 & 5,42 \\
$\begin{array}{c}\text { 2P-CON } \\
\text { OA }\end{array}$ & 2,86 & 2,19 & 2,14 & 7,18 \\
\hline
\end{tabular}

Cálculo de la media con OA

$\mathrm{N}=36$

Primer Parcial

$\mathrm{N} 1=$ rendimiento académico del 1P 
$\overleftarrow{\mathrm{N} 1}=\frac{\sum \mathrm{n} 1}{\mathrm{~N}}=\frac{195,11}{36}=5,42$

Segundo Parcial

$\mathrm{N} 2=$ rendimiento académico del $1 \mathrm{P}$

$\overleftarrow{\mathrm{N} 2}=\frac{\sum \mathrm{n} 2}{\mathrm{~N}}=\frac{258,54}{36}=7,18$

Matriz de registro de resultados de aprendizaje sin utilizar los Objetos de Aprendizaje.

Tabla No 4 Matriz de rendimiento académico SOA

\begin{tabular}{ccccc}
\hline & CD/4 & PAE/3 & AAA/3 & NOTA/10 \\
\hline $\begin{array}{c}\text { 1P-SIN } \\
\text { OA }\end{array}$ & 1,18 & 1,86 & 2,17 & 5,2 \\
$\begin{array}{c}\text { 2P-SIN } \\
\text { OA }\end{array}$ & 2,57 & 1,84 & 1,92 & 6,33 \\
\hline
\end{tabular}

Cálculo de la media sin OA

$\mathrm{N}=32$

Primer Parcial

$\mathrm{N} 1=$ rendimiento académico $=$ Nota final $1 \mathrm{P}$

$\overleftarrow{\mathrm{N} 1}=\frac{\sum \mathrm{n} 1}{\mathrm{~N}}=\frac{166,47}{32}=5,20$

Segundo Parcial

$\mathrm{N} 2=$ rendimiento académico $=$ Nota final del $2 \mathrm{P}$

$\overleftarrow{\mathrm{N} 2}=\frac{\sum \mathrm{n} 2}{\mathrm{~N}}=\frac{202,59}{32}=6,33$

Tabla No 5 comparación del acta final COA Y SOA

\begin{tabular}{lccc}
\hline & $\mathbf{N 1 / 1 0}$ & $\mathbf{N 2 / 1 0}$ & $\mathbf{N F} / 10$ \\
\hline CON OA & 5,42 & 9 & 7,21 \\
SIN OA & 5,2 & 6,3 & 5,8 \\
\hline
\end{tabular}




\section{Análisis estadístico descriptivo longitudinal con OA}

Tabla No 6 Comparación de las notas 1p-2p

\begin{tabular}{|c|c|c|c|c|}
\hline & P1 P2 & & $\mathrm{P} 1$ & $\mathrm{P} 2$ \\
\hline MIN & $3,6 \quad 0,0$ & MEDIA & 5,2 & 6,33 \\
\hline Q1 & 4,6 & MEDIANA & 5,2 & 7,00 \\
\hline MEDIANA & 5,2 & MODA & 5,0 & 7,00 \\
\hline Q3 & $5,7 \quad 7,7$ & DES ES & 0,8 & 2,25 \\
\hline
\end{tabular}

\begin{tabular}{lll}
\hline $\mathrm{MAX}$ & 78,5
\end{tabular}

\section{ACTA FINAL - OA}

P1 VS P2

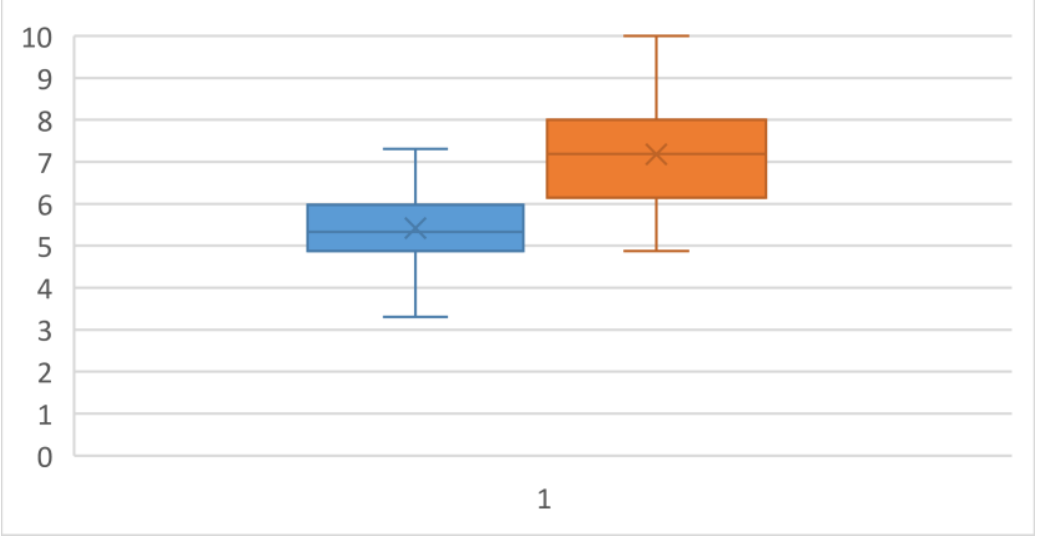

Fig. 1 Comparación de Actas Parciales con OA

Fuente: Elaboración propia

a) Interpretación. - Empleando los OA los estudiantes en el primer parcial alcanzan un rendimiento académico con un valor máximo atípico, en cambio en el segundo parcial alcanza 2 valores atípicos con respecto al mínimo y uno con respecto al máximo notándose una mejoría más centrada en el rendimiento académico muy satisfactorio. 


\section{Comparación de componentes N1-N2 del P1 y P2}

Tabla No 7 Comparación de actas de P1-P2 sin OA

\begin{tabular}{|c|c|c|c|c|c|}
\hline & $\bar{P} 1$ & $\mathrm{P} 2$ & & P1 & $\mathrm{P} 2$ \\
\hline MIN & 3,6 & 0,0 & MEDIA & 5,20 & 6,33 \\
\hline Q1 & 4,6 & 6 & MEDIANA & 5,20 & 7,00 \\
\hline MEDIANA & 5,2 & 7 & MODA & 5,00 & 7,00 \\
\hline Q3 & 5,725 & 7,7 & DES ES & 0,84 & 2,25 \\
\hline MAX & 7 & 8,50 & & & \\
\hline
\end{tabular}

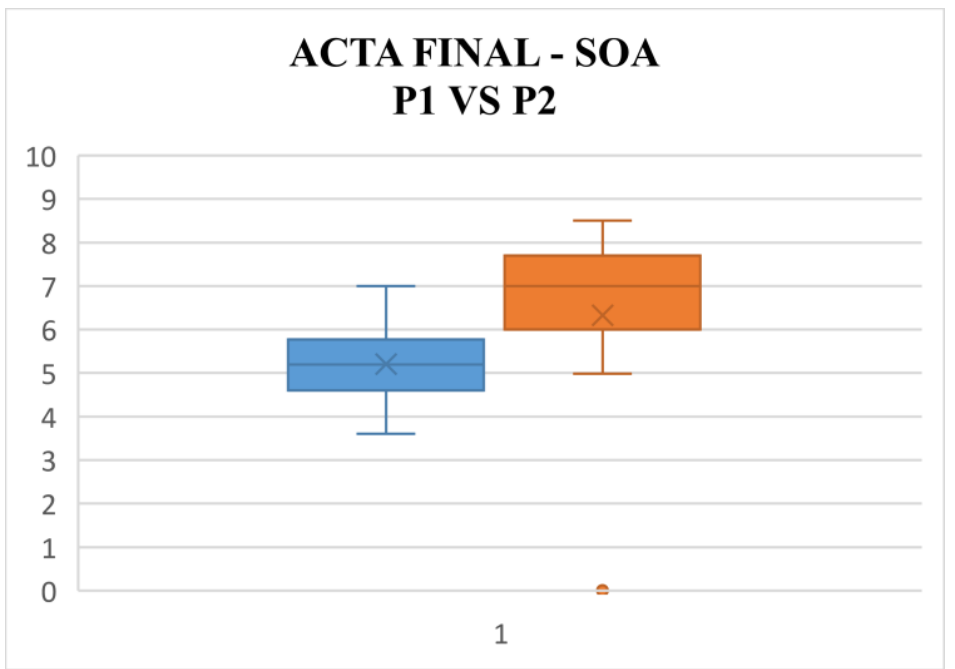

Fig. 1. Comparación de Actas Parciales sin OA

Fuente: Elaboración propia

a) Interpretación. - Del gráfico se puede deducir que la nota final del primer parcial su rendimiento académico es más consolidado pero menor, mientras en el segundo parcial muestra un rendimiento académico disperso, pero más alto.

Análisis estadístico transversal 
Comparación de Componentes de la nota Final 1P con OA - SOA

Tabla No 8 Comparación de N10A-N1SOA

\begin{tabular}{|c|c|c|c|c|}
\hline $\mathrm{N} 1 \mathrm{~F}$ & $\begin{array}{l}\text { N1- } \\
\text { COA }\end{array}$ & $\begin{array}{l}\text { N1- } \\
\text { SOA }\end{array}$ & $\mathrm{N} 1 \mathrm{~F}$ & $\begin{array}{cc}\text { N1- } & \text { N1- } \\
\text { COA } & \text { SOA }\end{array}$ \\
\hline MIN & 3,3 & 3,6 & MEDIA & $5,42 \quad 5,21$ \\
\hline Q1 & 4,8775 & 4,6 & MEDIANA & 5,33 \\
\hline MEDIANA & 5,33 & 5,2 & MODA & 5,50 \\
\hline Q3 & 5,8575 & 5,73 & DES ES & $\begin{array}{ll}0,89 & 0,84\end{array}$ \\
\hline MAX & 7,31 & 7 & & \\
\hline
\end{tabular}

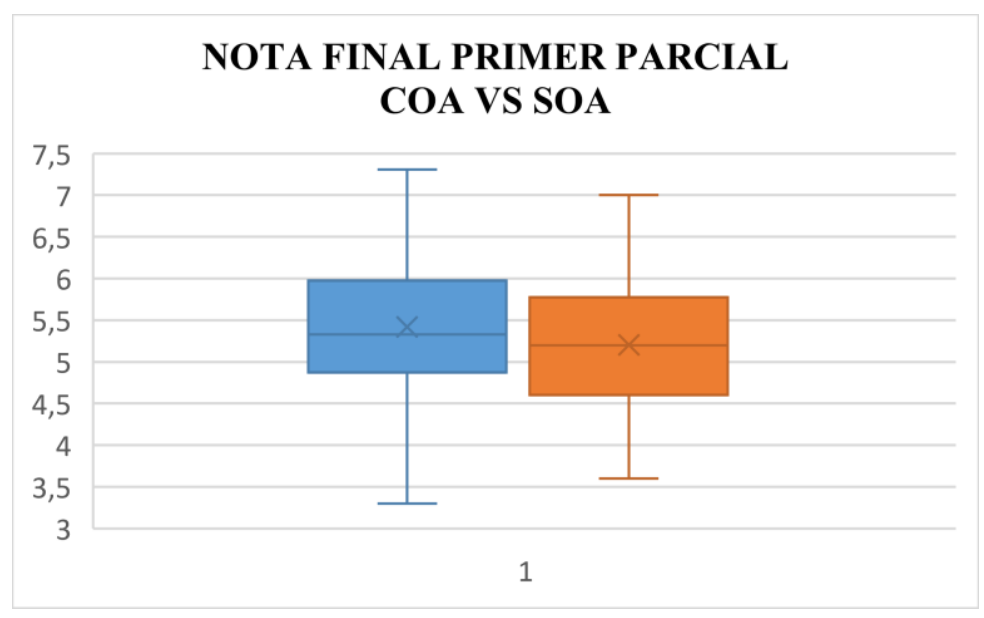

Fig. 3. 2 Gráfico de Comparación Actas Primer Parcial Fuente: Elaboración propia

a) Interpretación. -Del gráfico No. 15 la N1 del primer parcial con OA es más consolidado el rendimiento académico. En cambio, sin OA el rendimiento Académico es más disperso. 
Comparación de Componentes de Actas Finales COA - SOA

Tabla No 9 Comparación de notas del acta final

\begin{tabular}{|c|c|c|c|c|}
\hline $\mathrm{NF}$ & $\begin{array}{l}\text { NF- } \\
\mathrm{COA}\end{array}$ & $\begin{array}{l}\text { NF- } \\
\text { SOA }\end{array}$ & NF & $\begin{array}{ll}\text { NF- } & \text { NF- } \\
\text { COA } & \text { SOA }\end{array}$ \\
\hline$\overline{\text { MIN }}$ & 6 & 2 & $\overline{\text { MEDIA }}$ & $7,21 \quad 5,77$ \\
\hline Q1 & 6,93875 & 5,48375 & MEDIANA & $7,17 \quad 6,20$ \\
\hline MEDIANA & 7,165 & 6,2 & $\overline{M O D A}$ & $7,25 \quad 6,55$ \\
\hline Q3 & 7,165 & 6,2 & DES ES & $0,45 \quad 1,26$ \\
\hline$\overline{M A X}$ & 8 & 7 & & \\
\hline
\end{tabular}

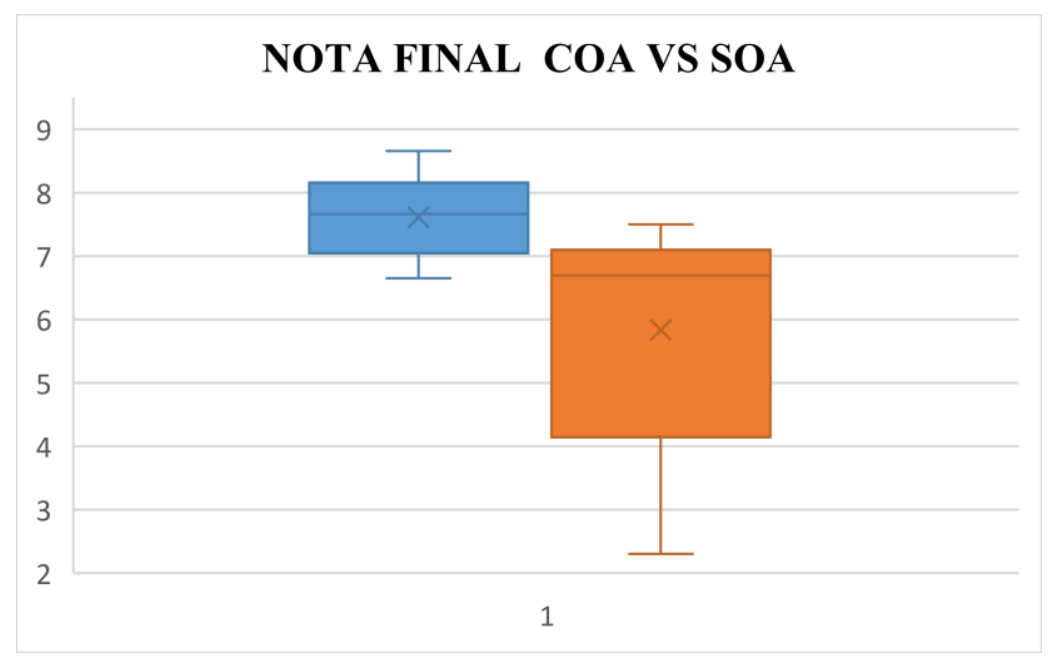

Fig. 4. Comparación de nota final COA y SOA

Fuente: Elaboración propia

a) Interpretación. - Del gráfico No.20 se puede apreciar que el rendimiento académico con OA es más conciso, mientras que el rendimiento académico sin OA es muy disperso. 


\section{Conclusiones.}

- La experiencia realizada demuestra que es posible elevar el nivel de rendimiento académico en la asignatura de Física I, lo resultados obtenidos de nuestra experiencia demuestra la posibilidad, cuando forma parte de los objetivos y están dirigidos con un propósito.

- Se construyó OA para facilitar el proceso de enseñanza y aprendizaje de la signatura de Física I en el estudio de la Dinámica de la partícula, motivando de esta forma a docentes que incursionen en esta nueva temática educativa.

- Se incorporó los OA en la enseñanza de la Física I para motivar al estudiante a la forma de trabajar de modo independiente y colectivo mediante el intercambio de experiencias entre compañeros con actividades específicas.

- Los OA permitirán potencializar la educación, ya que ofrecen la posibilidad de tener contenidos educativos reutilizables, independientes de la plataforma de uso.

- Los OA facilitará al docente elaborar sílabos más flexibles que se adapten a las necesidades específicas de cómo perfeccionar el rendimiento académico en el estudiante.

\section{Referencias bibliográficas.}

Aldana, S., Vereda, F., Hidalgo-Alvarez, R., \& de Vicente, J. (2016). Facile synthesis of magnetic agarose microfibers by directed selfassembly. Polymer, 93, 61-64.

Allport, G. (16 de 05 de 2016). Psicología online. Obtenido de http://www.psicologiaonline.com/ebooks/personalidad/allport.htm: http://www.psicologiaonline.com/ebooks/personalidad/allport.htm

Antueno, Eduardo A. . (2008). Simulaciones para la enseñanza de física en la universidad. Buenos Aires, Argentina.

Callejas, M. H. (2011). Objetos de aprendizaje , u estado del arte. Mexico: Redalyc.

Castiblanco, O. y. (2008). El uso de las TIC's en la enseñanza de la Física. Académico, 7.

Covington, M., \& Omelich, C. (1979). ¿Are causal attribution causal?: A path analysis of cognitive model achievement motivation. Journal of Persanality and Social Psychology,37, págs. 1487-1504.

Covington, M., \& Omelich, C. (1979). A path analysis of the cognitive model of archievement motivation. Journal of Personality and Social Psychology, 37, 14871504.

Davidov, V. e. (1991). La enseñanza que Desarrolla en la escuela del Desarrollo; en La educación y la enseñanza: una mirada al futuro. Moscu: Progresso.

Diaz, F. (2002). estrategias docentes para un aprendizaje significativo. Mexico: Mc Graw Hill.

Fernández, Y. (2011). Variables académicas que influyen en el Rendimiento Académico de los Estudiantes Universitarios. Ivestigacion Educativa Vol15 No 27 165-179 EneroJunio 2011 ISSN1728-5852, 15. 
Ferreyra, H. (24 de julio de 2015). Wikipedia. Obtenido de https://es.wikipedia.org/wiki/Aprendizaje_significativo

Garbanzo Vargas, G. (1997). "Factores asociados al rendimiento académico en los estudios universitarios, una reflexión desde la calidda de la educación superior". Costa Rica: Universidad de Costa Rica.

Garbanzo, G. (2007). factores ascociados al Rendimiento Aacdémico en estudiantes Universitarios. Revista Educación , 43-63.

García-Cerda, L., Rodríguez-Fernández, O., Betancourt-Galindo, R., Saldívar-Guerrero, R., \& Torres-Torres, M. (2003). Síntesis y propiedades de ferrofluidos de magnetita. Superficies y Vacío., 16(1), 28-31.

Hodgins, W. (1992). Los objetos de aprendizaje digitales. Obtenido de http://www.ehistoria.cl/e-historia/los-objetos-digitales-de-aprendizaje-odas-2/

http://constructivismo.webnode.es/rss/. (11 de abril de 2016). Obtenido de http://constructivismo.webnode.es/rss/: www.constructivismo.webmode.es

https://profesiondocente. wikispaces.com/Modelo+de+evaluaci\%C3\%B3n+de+Guskey. (6 $\begin{array}{lllll}\text { de } & 05 & \text { de } & \text { Obtenido }\end{array}$ https://profesiondocente.wikispaces.com/Modelo+de+evaluaci\%C3\%B3n+de+Gusk ey:

https://profesiondocente.wikispaces.com/Modelo+de+evaluaci\%C3\%B3n+de+Gusk ey

Loza, C., Guffante, T., Murillo, M., Tenesaca, R., Montalvo, C., Garcia , C., . . López, E. (2014). Modelo educativo. Universidad Nacional de Chimborazo, Unidad de Planificación Académica, Riobamba.

Monografías. (26 de 06 de 2016). http://www.monografias.com/trabajos58/principales-tiposinvestigacion/principales-tipos-investigacion.shtml. Obtenido de http://www.monografias.com/trabajos10/inin/inin.shtml

Moreno Escobar, J. (2005). Metodología para la creación de Objetos de aprendizaje de apoyo a la Educación. Escuela Superior de Ingeniería Mecánica y Eléctrica. Mexico: Instituto Politécnico Nacional.

Orozco, Y. R. (2015). La enseñanza de la Física mediante Objetos de Aprendizaje. Revista de Ciencias Pedagógicas, 10.

Pelaez, C., \& Wilmer, J. (2001). Análisis multivariante para medir el rendimiento académico de los estudiates de una carrera universitaria. Guayaquil: dspace.espol.edu.ec.

Ramos Ortega, O. (2015). Elaboración y aplicación de una guía metodológica Interactive Physics. En el Rensdimiento Académico de Cinemática de los estudiantes de primer Año de bachillerato del Colegio Teécnico Nacional "Miguel Angel León Pontón" periodo primer tremestre. Riobamba: $\mathrm{SN}$.

Ramos, O. (2015). Elaboración y aplicación de una guía metodológica de interactive physics. Riobamba: S/N.

Ruiz Estrada, G. (2004). Desarrollo de un Sistema de liberación de fármacos basado en nanopartículas magnéticas recubiertas con Polietilénglicol para el tratamiento de diferentes enfermedades. Madrid: Universidad Autónoma de Madrid. Departamento de Física Aplicada.

Ruiz, J. (2005). Alternativa metodológica para la formación integral de los estudiantes desde el proceso de enseñanza- aprendizaje de la Física. Camaguey: Centro de estudios de Ciencias de la Educación "Enrique José Varona". 
Santillana. (2009). ¿Cómo hcer el aprendizaje significatico? Ecuador: Mariscal.

Senescyt. (2013). Micro currículo de la Física para Ciencias e Ingeniería-Senescyt 2013. Quito.

Squires, D., \& McDougall, A. (1997). Como elegir y utilizar software educativo. Madrid: Ediciones Morata.

Tejedor, f. y. (2007). Causas del bajo REendimiento del estudiante universitario. Revista de Educación, 443-473.

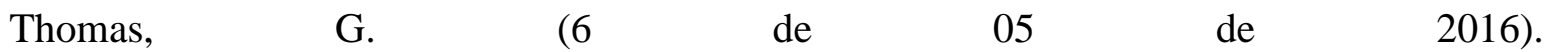
https://profesiondocente.wikispaces.com/Modelo+de+evaluaci\%C3\%B3n+de+Gusk ey. Obtenido de https://profesiondocente.wikispaces.com/Modelo+de+evaluaci\%C3\%B3n+de+Gusk ey:

https://profesiondocente.wikispaces.com/Modelo+de+evaluaci\%C3\%B3n+de+Gusk ey

Turoff, M. (1995). http://www.njit.edu/njiT/Department/CCCC/VC/Papers/Design.html. Design Virtual Clasroom. Obtenido de http://www.njit.edu/njiT/Department/CCCC/VC/Papers/Design.html: http://www.njit.edu/njiT/Department/CCCC/VC/Papers/Design.html

Unidad de planificación académica. (2014). Modelo Educativo, Pedagógico y Didáctico. Riobamba: UNACH.

Urquizo, A. (2005). Como realizar la tesis o una investigación. Riobamba, Ecuador: Gráficas Riobamba.

Wiley, D. (2000). Connecting learning objetcs to instructional design theory: A definition a metaphor, and taxonomy. Obtenido de Connecting learning objetcs to instructional design theory: A definition a metaphor, and taxonomy: http://reausability.org/read/chapters/wiley.doc.

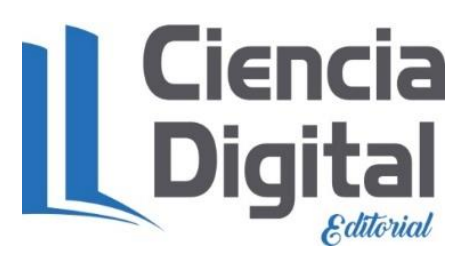


Para citar el artículo indexado.

Martinez Nogales, J., Cachuput Gusñay, J., Guilcapi Mosquera, J., \& Carrasco Pilco, L. (2019). Utilización de los objetos de aprendizaje y su relación en el rendimiento académico de la asignatura $\begin{array}{lllll}\text { de Física } & \text { I. } & \text { Explorador } & \text { 105-122. }\end{array}$ https://doi.org/10.33262/exploradordigital.v3i3.1.870

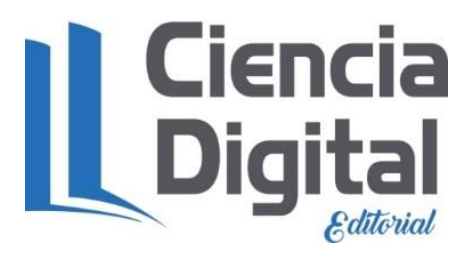

El artículo que se publica es de exclusiva responsabilidad de los autores y no necesariamente reflejan el pensamiento de la Revista Explorador Digital.

El articulo queda en propiedad de la revista y, por tanto, su publicación parcial y/o total en otro medio tiene que ser autorizado por el director o editor de la Revista Explorador

\section{Digital.}
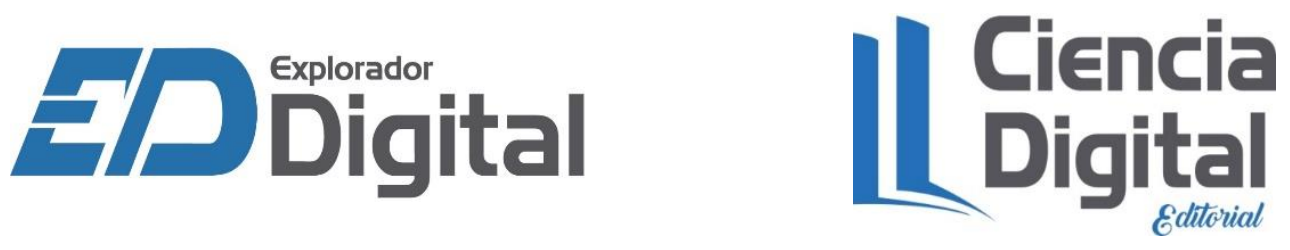
